\title{
Quantum discord and multipartite correlations
}

\author{
Małgorzata Okrasa* and Zbigniew Walczal $\mathbb{A}^{\oplus}$ \\ Department of Theoretical Physics, University of Lodz \\ Pomorska 149/153, 90-236 Eódź, Poland, EU
}

(Dated: November 13, 2018)

\begin{abstract}
Recently, it was realized that quantum discord can be seen as the minimal amount of correlations which are lost when some local quantum operations are performed. Based on this formulation of quantum discord, we provide a systematical analysis of quantum and classical correlations present in both bipartite and multipartite quantum systems. As a natural result of this analysis, we introduce a new measure of the overall quantum correlations which is lower bounded by quantum discord.
\end{abstract}

PACS numbers: 03.67.-a, 03.65.-w

\section{INTRODUCTION}

In quantum information theory, the problem of characterization of correlations present in a quantum state has been intensively studied during the last two decades (for review, see [1, 2]). The most significant progress has been made in this subject in the case of bipartite quantum systems, especially low-dimensional ones, which have been studied in the framework of paradigm based on the entanglement-separability dichotomy introduced by Werner [3]. In particular, in the framework of this approach it has become clear that the correlations present in a quantum state can be classified as either classical or quantum, where the latter ones cannot exist without the former ones which are identified with entanglement. However, some results showed that quantum correlations cannot be only limited to entanglement, because separable quantum states can also have correlations which are responsible for the improvements of some quantum tasks that cannot be simulated by classical methods [4 10]. Therefore, there is a need to study correlations from a perspective different than the entanglement-separability paradigm.

The first attempt in this direction was made by Ollivier and Zurek [11] who studied quantum correlations from a measurement perspective. They considered two natural quantum extensions of the classical mutual information and showed that their difference, called quantum discord, can be used as a measure of the quantumness of correlations in bipartite quantum states, including separable ones. Alternative but closely related attempt in going beyond the entanglement-separability paradigm was made independently by Henderson and Vedral [12] who tried to separate classical and quantum correlations in bipartite quantum states.

Quantum discord became a subject of intensive study in different contexts after the recent discovery $13-15$ that non-classical correlations other than entanglement can be responsible for the quantum computational effi-

\footnotetext{
*Electronic address: okrasa@merlin.phys.uni.lodz.pl
}

${ }^{\dagger}$ Electronic address: walczak@merlin.phys.uni.lodz.pl ciency of deterministic quantum computation with one pure qubit [4]. Because evaluation of quantum discord involves optimization procedure, it was analytically computed only for a few families of two-qubit states [16 18]. In these cases, examination of the structure of entanglement and discord showed that quantum discord is a measure of non-classical correlations that may include entanglement however, discord is an independent measure.

Moreover, when Markovian and non-Markovian dynamics of discord was analyzed [19 23], it was discovered that quantum discord and entanglement can behave very differently - in contrast with entanglement, in considered cases, Markovian evolution can never lead to a sudden death of discord, while non-Markovian can lead to its sudden birth.

In the context of complete positivity of reduced quantum dynamics, it was discovered that an arbitrary unitary evolution for any system and environment is described as a completely positive map on the system iff system and environment are initially in a zero-discord state [24, 25]. Furthermore, it was shown that only some zero-discord states can be locally broadcast [26]. Remarkably, it was discovered that a random quantum state possesses in general strictly positive discord and an arbitrarily small perturbation of a zero-discord state will generate discord - in other words zero-discord states are extremely rare [27]. Recently, a necessary and sufficient condition for the existence of non-zero quantum discord was obtained [28]. Furthermore, a natural witness for quantum discord for $2 \times N$ states was provided 29]. Moreover, the notion of quantum discord was also extended to continuous variable systems to study correlations in two-mode Gaussian states [30, 31].

In this article, we provide a systematical analysis of quantum and classical correlations present in bipartite quantum systems using an alternative formulation of quantum discord. As a natural result of this analysis, we introduce a new measure of the overall bipartite quantum correlations and we show that this measure is lower bounded by quantum discord. Finally, we generalize a notion of quantum discord to multipartite quantum systems, by invoking quantum relative entropy, and then we show that our approach to quantification of correlations can be naturally extended to multipartite quantum 
systems.

\section{QUANTUM DISCORD IN BIPARTITE SYSTEMS}

Let us consider two quantum systems, $A$ and $B$, in a state $\rho_{A B}$. In quantum information theory, the quantum mutual information of a state $\rho_{A B}$,

$$
\mathcal{I}\left(\rho_{A B}\right)=S\left(\rho_{A}\right)+S\left(\rho_{B}\right)-S\left(\rho_{A B}\right),
$$

is regarded as a measure of the total correlations (classical and quantum) present in a state $\rho_{A B}$, where $\rho_{A(B)}$ is the reduced state of the system $A(B)$, and $S(\rho)=$ $-\operatorname{Tr}\left(\rho \log _{2} \rho\right)$ is the von Neumann entropy. The quantum conditional entropy, $S\left(\rho_{B \mid A}\right)=S\left(\rho_{A B}\right)-S\left(\rho_{A}\right)$, allows one to rewrite the quantum mutual information in the following form

$$
\mathcal{I}\left(\rho_{A B}\right)=S\left(\rho_{B}\right)-S\left(\rho_{B \mid A}\right) .
$$

The fact that the quantum conditional entropy quantifies the ignorance about the system $B$ that remains if we make measurements on the system $A$ allows one to find an alternative expression for the quantum conditional entropy, and thereby for the quantum mutual information.

If the von Neumann projective measurement, described by a complete set of one-dimensional orthogonal projectors, $\left\{\Pi_{i}^{A}\right\}$, corresponding to outcomes $i$, is performed, then the state of the system $B$ after the measurement is given by $\rho_{B \mid i}=\operatorname{Tr}_{A}\left[\left(\Pi_{i}^{A} \otimes I\right) \rho_{A B}\left(\Pi_{i}^{A} \otimes I\right)\right] / p_{i}^{A}$, where $p_{i}^{A}=\operatorname{Tr}\left[\left(\Pi_{i}^{A} \otimes I\right) \rho_{A B}\right]$. The von Neumann entropies $S\left(\rho_{B \mid i}\right)$, weighted by probabilities $p_{i}^{A}$, lead to the quantum conditional entropy of the system $B$ given the complete measurement $\left\{\Pi_{i}^{A}\right\}$ on the system $A$

$$
S_{\left\{\Pi_{i}^{A}\right\}}\left(\rho_{B \mid A}\right)=\sum_{i} p_{i}^{A} S\left(\rho_{B \mid i}\right),
$$

and thereby the quantum mutual information, induced by the von Neumann measurement performed on the system $A$, is defined by $\mathcal{J}_{\left\{\Pi_{i}^{A}\right\}}\left(\rho_{A B}\right)=S\left(\rho_{B}\right)-S_{\left\{\Pi_{i}^{A}\right\}}\left(\rho_{B \mid A}\right)$. The measurement independent quantum mutual information $\mathcal{J}_{A}\left(\rho_{A B}\right)$, defined by

$$
\begin{aligned}
\mathcal{J}_{A}\left(\rho_{A B}\right) & =\sup _{\left\{\Pi_{i}^{A}\right\}} \mathcal{J}_{\left\{\Pi_{i}^{A}\right\}}\left(\rho_{A B}\right) \\
& =S\left(\rho_{B}\right)-\inf _{\left\{\Pi_{i}^{A}\right\}} \sum_{i} p_{i}^{A} S\left(\rho_{B \mid i}\right),
\end{aligned}
$$

is interpreted as a measure of classical correlations, $\mathcal{C}_{A}\left(\rho_{A B}\right)=\mathcal{J}_{A}\left(\rho_{A B}\right)\left[11,12\right.$. In general case, $\mathcal{I}\left(\rho_{A B}\right)$ and $\mathcal{J}_{A}\left(\rho_{A B}\right)$ may differ and the difference which is interpreted as a measure of quantum correlations,

$$
\begin{aligned}
\mathcal{D}_{A}\left(\rho_{A B}\right) & =\mathcal{I}\left(\rho_{A B}\right)-\mathcal{C}_{A}\left(\rho_{A B}\right) \\
& =S\left(\rho_{A}\right)-S\left(\rho_{A B}\right)+\inf _{\left\{\Pi_{i}^{A}\right\}} \sum_{i} p_{i}^{A} S\left(\rho_{B \mid i}\right)
\end{aligned}
$$

is called quantum discord [1]]. It is obvious that, in general, the quantum discord $\mathcal{D}_{A}\left(\rho_{A B}\right)$ is not symmetric with respect to the systems $A$ and $B$. However, swapping a role of $A$ and $B$ one can easily get

$$
\begin{aligned}
\mathcal{D}_{B}\left(\rho_{A B}\right) & =\mathcal{I}\left(\rho_{A B}\right)-\mathcal{C}_{B}\left(\rho_{A B}\right) \\
& =S\left(\rho_{B}\right)-S\left(\rho_{A B}\right)+\inf _{\left\{\Pi_{j}^{B}\right\}} \sum_{j} p_{j}^{B} S\left(\rho_{A \mid j}\right),
\end{aligned}
$$

where now the von Neumann projective measurement, described by a complete set of one-dimensional orthogonal projectors, $\left\{\Pi_{j}^{B}\right\}$, corresponding to outcomes $j$, is performed on the system $B$, and the state of the system $A$ after the measurement is given by $\rho_{A \mid j}=\operatorname{Tr}_{B}[(I \otimes$ $\left.\left.\Pi_{j}^{B}\right) \rho_{A B}\left(I \otimes \Pi_{j}^{B}\right)\right] / p_{j}^{B}$, where $p_{j}^{B}=\operatorname{Tr}\left[\left(I \otimes \Pi_{j}^{B}\right) \rho_{A B}\right]$.

\section{CORRELATIONS IN BIPARTITE SYSTEMS}

Recently, it was realized that the quantum discord $\mathcal{D}_{A}\left(\rho_{A B}\right)$ can be expressed alternatively as the minimal loss of correlations caused by the non-selective von Neumann projective measurement performed on the system A 32 ]

$$
\mathcal{D}_{A}\left(\rho_{A B}\right)=\inf _{\left\{\Pi_{i}^{A}\right\}}\left[\mathcal{I}\left(\rho_{A B}\right)-\mathcal{I}\left(\mathcal{M}_{\left\{\Pi_{i}^{A}\right\}}\left(\rho_{A B}\right)\right)\right],
$$

where $\mathcal{M}_{\left\{\Pi_{i}^{A}\right\}}\left(\rho_{A B}\right)=\sum_{i}\left(\Pi_{i}^{A} \otimes I\right) \rho_{A B}\left(\Pi_{i}^{A} \otimes I\right)$.

In this section, we will explain first why this formulation of quantum discord is equivalent to its original definition given by equations (5). Then, using this formulation of quantum discord we will investigate correlations present in bipartite systems.

According to the quantum operations formalism 33, 34] the most general transformation of a quantum state $\rho$ can be represented by a linear, completely positive, trace-preserving map $\mathcal{E}$. A quantum operation $\mathcal{E}$ can be written in a form known as the operator-sum representation $\mathcal{E}(\rho)=\sum_{i} E_{i} \rho E_{i}^{\dagger}$ where operation elements $\left\{E_{i}\right\}$, called the Kraus operators, satisfy the completeness relation $\sum_{i} E_{i}^{\dagger} E_{i}=I$.

Therefore, we see that from the viewpoint of quantum operations formalism, the non-selective von Neumann projective measurement performed on the system $A$ is a local quantum operation, $\mathcal{M}_{\left\{\Pi_{i}^{A}\right\}}$, with operation elements $\left\{\Pi_{i}^{A} \otimes I\right\}$.

Since the quantum mutual information $\mathcal{I}\left(\rho_{A B}\right)$ does not increase under local quantum operations [34], therefore the difference $\mathcal{I}\left(\rho_{A B}\right)-\mathcal{I}\left(\mathcal{M}_{\left\{\Pi_{i}^{A}\right\}}\left(\rho_{A B}\right)\right)$ describes the correlations loss under local quantum operation $\mathcal{M}_{\left\{\Pi_{i}^{A}\right\}}$.

Let us note that the joint state of systems $A$ and $B$ after performing the non-selective von Neumann projective measurement on the system $A$ is given by

$$
\mathcal{M}_{\left\{\Pi_{i}^{A}\right\}}\left(\rho_{A B}\right)=\sum_{i} p_{i}^{A} \Pi_{i}^{A} \otimes \rho_{B \mid i}
$$


whereas the following equations describe the state of system $A$ and $B$, respectively

$$
\begin{aligned}
& \operatorname{Tr}_{B}\left[\mathcal{M}_{\left\{\Pi_{i}^{A}\right\}}\left(\rho_{A B}\right)\right]=\sum_{i} p_{i}^{A} \Pi_{i}^{A}, \\
& \operatorname{Tr}_{A}\left[\mathcal{M}_{\left\{\Pi_{i}^{A}\right\}}\left(\rho_{A B}\right)\right]=\sum_{i} p_{i}^{A} \rho_{B \mid i}=\rho_{B} .
\end{aligned}
$$

Using the elementary properties of the von Neumann entropy, $S\left(\sum_{i} p_{i}^{A} \Pi_{i}^{A} \otimes \rho_{B \mid i}\right)=H\left(p_{i}^{A}\right)+\sum_{i} p_{i}^{A} S\left(\rho_{B \mid i}\right)$ and $S\left(\sum_{i} p_{i}^{A} \Pi_{i}^{A}\right)=H\left(p_{i}^{A}\right)$ 34], where $H\left(p_{i}^{A}\right)=$ $-\sum_{i} p_{i}^{A} \log _{2} p_{i}^{A}$ is the Shannon entropy, we can now compute the quantum mutual information of $\mathcal{M}_{\left\{\Pi_{i}^{A}\right\}}\left(\rho_{A B}\right)$ via equations (11), (8) and (9)

$$
\begin{aligned}
\mathcal{I}\left(\mathcal{M}_{\left\{\Pi_{i}^{A}\right\}}\left(\rho_{A B}\right)\right) & =S\left(\rho_{B}\right)-\sum_{i} p_{i}^{A} S\left(\rho_{B \mid i}\right) \\
& =\mathcal{J}_{\left\{\Pi_{i}^{A}\right\}}\left(\rho_{A B}\right) .
\end{aligned}
$$

Therefore, the correlations loss under local quantum operation $\mathcal{M}_{\left\{\Pi_{i}^{A}\right\}}, \mathcal{I}\left(\rho_{A B}\right)-\mathcal{I}\left(\mathcal{M}_{\left\{\Pi_{i}^{A}\right\}}\left(\rho_{A B}\right)\right)$, is equal to $\mathcal{I}\left(\rho_{A B}\right)-\mathcal{J}_{\left\{\Pi_{i}^{A}\right\}}\left(\rho_{A B}\right)$. Consequently, the minimal loss of correlations caused by local quantum operation $\mathcal{M}_{\left\{\Pi_{i}^{A}\right\}}$ is given by

$$
\begin{aligned}
& \inf _{\left\{\Pi_{i}^{A}\right\}}\left[\mathcal{I}\left(\rho_{A B}\right)-\mathcal{I}\left(\mathcal{M}_{\left\{\Pi_{i}^{A}\right\}}\left(\rho_{A B}\right)\right)\right] \\
& =\mathcal{I}\left(\rho_{A B}\right)-\sup _{\left\{\Pi_{i}^{A}\right\}} \mathcal{J}_{\left\{\Pi_{i}^{A}\right\}}\left(\rho_{A B}\right) \\
& =\mathcal{I}\left(\rho_{A B}\right)-\mathcal{C}_{A}\left(\rho_{A B}\right)=\mathcal{D}_{A}\left(\rho_{A B}\right) .
\end{aligned}
$$

This shows that quantum correlations present in a bipartite state $\rho_{A B}$, as measured by $\mathcal{D}_{A}\left(\rho_{A B}\right)$, can be seen as the minimal amount of correlations which are lost when the non-selective von Neumann projective measurement is performed on the system $A$.

Let us note that performing the optimal non-selective von Neumann projective measurement $\mathcal{M}_{\left\{\widetilde{\Pi}_{i}^{A}\right\}}$, for which supremum in equation (11b) is attained, we leave classical correlations unaffected, because $\mathcal{D}_{A}\left(\mathcal{M}_{\left\{\widetilde{\Pi}_{i}^{A}\right\}}\left(\rho_{A B}\right)\right)=$ 0 [35] which implies via equations (11) that

$$
\mathcal{C}_{A}\left(\mathcal{M}_{\left\{\widetilde{\Pi}_{i}^{A}\right\}}\left(\rho_{A B}\right)\right)=\mathcal{I}\left(\mathcal{M}_{\left\{\widetilde{\Pi}_{i}^{A}\right\}}\left(\rho_{A B}\right)\right)=\mathcal{C}_{A}\left(\rho_{A B}\right) .
$$

Although the measurement $\mathcal{M}_{\left\{\widetilde{\Pi}_{i}^{A}\right\}}$ causes only the loss of quantum correlations in the state $\rho_{A B}$, according to classification of bipartite quantum states [26] the state

$$
\mathcal{M}_{\left\{\widetilde{\Pi}_{i}^{A}\right\}}\left(\rho_{A B}\right)=\sum_{i} \tilde{p}_{i}^{A} \widetilde{\Pi}_{i}^{A} \otimes \rho_{B \mid i}
$$

can have quantum correlations, which are not captured by $\mathcal{D}_{A}\left(\mathcal{M}_{\left\{\widetilde{\Pi}_{i}^{A}\right\}}\left(\rho_{A B}\right)\right)$, because the states $\rho_{B \mid i}$ do not necessarily commute -according to classification of bipartite quantum states [26], if the states $\rho_{B \mid i}$ commute, then the state (13) has only classical correlations, otherwise the state (13) has classical and quantum correlations.
In order to investigate quantum correlations present in the state $\mathcal{M}_{\left\{\widetilde{\Pi}_{i}^{A}\right\}}\left(\rho_{A B}\right)$, let us note that quantum discord $\mathcal{D}_{B}\left(\rho_{A B}\right)$ can be expressed alternatively as the minimal loss of correlations caused by the non-selective von Neumann projective measurement performed on the system $B$

$$
\begin{aligned}
& \mathcal{D}_{B}\left(\rho_{A B}\right)=\inf _{\left\{\Pi_{j}^{B}\right\}}\left[\mathcal{I}\left(\rho_{A B}\right)-\mathcal{I}\left(\mathcal{M}_{\left\{\Pi_{j}^{B}\right\}}\left(\rho_{A B}\right)\right)\right] \\
& =\mathcal{I}\left(\rho_{A B}\right)-\sup _{\left\{\Pi_{j}^{B}\right\}} \mathcal{J}_{\left\{\Pi_{j}^{B}\right\}}\left(\rho_{A B}\right) \\
& =\mathcal{I}\left(\rho_{A B}\right)-\mathcal{C}_{B}\left(\rho_{A B}\right)
\end{aligned}
$$

where $\mathcal{M}_{\left\{\Pi_{j}^{B}\right\}}\left(\rho_{A B}\right)=\sum_{j}\left(I \otimes \Pi_{j}^{B}\right) \rho_{A B}\left(I \otimes \Pi_{j}^{B}\right)$.

It is clear that when we perform the optimal nonselective von Neumann projective measurement $\mathcal{M}_{\left\{\tilde{\Pi}_{j}^{B}\right\}}$, for which supremum in equation (14b) is attained, then the post-measurement joint state is given by

$$
\mathcal{M}_{\left\{\widetilde{\Pi}_{j}^{B}\right\}}\left(\mathcal{M}_{\left\{\widetilde{\Pi}_{i}^{A}\right\}}\left(\rho_{A B}\right)\right)=\sum_{i j} \tilde{p}_{i j}^{A B} \widetilde{\Pi}_{i}^{A} \otimes \widetilde{\Pi}_{j}^{B},
$$

where $\tilde{p}_{i j}^{A B}=\operatorname{Tr}\left[\left(\widetilde{\Pi}_{i}^{A} \otimes \widetilde{\Pi}_{j}^{B}\right) \rho_{A B}\right]$. Let us note that performing this measurement, we leave classical correlations unaffected, because $\mathcal{D}_{B}\left(\mathcal{M}_{\left\{\widetilde{\Pi}_{j}^{B}\right\}}\left(\mathcal{M}_{\left\{\widetilde{\Pi}_{i}^{A}\right\}}\left(\rho_{A B}\right)\right)\right)=0$ [35] which implies via equations (14) that

$$
\begin{aligned}
\mathcal{C}_{B}\left(\mathcal{M}_{\left\{\widetilde{\Pi}_{j}^{B}\right\}}\left(\mathcal{M}_{\left\{\widetilde{\Pi}_{i}^{A}\right\}}\left(\rho_{A B}\right)\right)\right) & =\mathcal{I}\left(\mathcal{M}_{\left\{\widetilde{\Pi}_{j}^{B}\right\}}\left(\mathcal{M}_{\left\{\widetilde{\Pi}_{i}^{A}\right\}}\left(\rho_{A B}\right)\right)\right) \\
& =\mathcal{C}_{B}\left(\mathcal{M}_{\left\{\widetilde{\Pi}_{i}^{A}\right\}}\left(\rho_{A B}\right)\right), \quad(16)
\end{aligned}
$$

where equations (14) were applied to the state $\mathcal{M}_{\left\{\widetilde{\Pi}_{i}^{A}\right\}}\left(\rho_{A B}\right)$ instead of $\rho_{A B}$. The above considerations show that the measurement $\mathcal{M}_{\left\{\widetilde{\Pi}_{j}^{B}\right\}}$ causes only the loss of quantum correlations in the state $\mathcal{M}_{\left\{\widetilde{\Pi}_{i}^{A}\right\}}\left(\rho_{A B}\right)$. According to classification of bipartite quantum states [26], the resulting state (15) has only classical correlations.

Since we have shown that the subsequent optimal measurements $\mathcal{M}_{\left\{\widetilde{\Pi}_{i}^{A}\right\}}$ and $\mathcal{M}_{\left\{\widetilde{\Pi}_{j}^{B}\right\}}$ performed on systems $A$ and $B$, respectively, lead only to the loss of all quantum correlations leaving classical correlations unaffected, and since we know exaclty the amount of quantum correlations which are lost when the optimal local measurements are performed, we can introduce, in a natural way, a new measure of the overall quantum correlations present in a bipartite state $\rho_{A B}$ which is based on quantum discord

$$
Q\left(\rho_{A B}\right)=\mathcal{D}_{A}\left(\rho_{A B}\right)+\mathcal{D}_{B}\left(\mathcal{M}_{\left\{\widetilde{\Pi}_{i}^{A}\right\}}\left(\rho_{A B}\right)\right) .
$$

As an illustrative simple example, let us consider two qubits in the state $|\psi\rangle_{A B}=(|0\rangle|0\rangle+|1\rangle|+\rangle) / \sqrt{2}$, where $|+\rangle=(|0\rangle+|1\rangle) / \sqrt{2}$. It can be verified that $\mathcal{D}_{A}\left(\rho_{A B}\right)=$ $2-\frac{1}{4}\left[(2+\sqrt{2}) \log _{2}(2+\sqrt{2})+(2-\sqrt{2}) \log _{2}(2-\sqrt{2})\right] \simeq$ 0.600876 , and the optimal measurement $\mathcal{M}_{\left\{\widetilde{\Pi}_{i}^{A}\right\}}$ is described by $\widetilde{\Pi}_{0}^{A}=|0\rangle\langle 0|$ and $\widetilde{\Pi}_{1}^{A}=|1\rangle\langle 1|$. Let us note that the post-measurement state $\mathcal{M}_{\left\{\widetilde{\Pi}_{i}^{A}\right\}}\left(\rho_{A B}\right)=$ 
$\frac{1}{2}(|0\rangle\langle 0|\otimes| 0\rangle\langle 0|+| 1\rangle\langle 1|\otimes|+\rangle\langle+|)$ has quantum correlations. It can be verified that $\mathcal{D}_{B}\left(\mathcal{M}_{\left\{\widetilde{\Pi}_{i}^{A}\right\}}\left(\rho_{A B}\right)\right)=$ $3-\frac{1}{2}\left[(2+\sqrt{2}) \log _{2}(2+\sqrt{2})+(2-\sqrt{2}) \log _{2}(2-\sqrt{2})\right] \simeq$ 0.201752 , and the optimal measurement $\mathcal{M}_{\left\{\widetilde{\Pi}_{j}^{B}\right\}}$ is described by $\widetilde{\Pi}_{0}^{B}=\left(\sin \frac{\pi}{8}|0\rangle+\cos \frac{\pi}{8}|1\rangle\right)\left(\sin \frac{\pi}{8}\langle 0|+\cos \frac{\pi}{8}\langle 1|\right)$ and $\widetilde{\Pi}_{1}^{B}=\left(\cos \frac{\pi}{8}|0\rangle-\sin \frac{\pi}{8}|1\rangle\right)\left(\cos \frac{\pi}{8}\langle 0|-\sin \frac{\pi}{8}\langle 1|\right)$. Since the subsequent optimal measurements performed on systems $A$ and $B$, respectively lead only to the loss of all quantum correlations leaving classical correlations unaffected, thus we see that the overall quantum correlations present in the state $|\psi\rangle_{A B}$ are quantified by $Q\left(\rho_{A B}\right)=$ $\mathcal{D}_{A}\left(\rho_{A B}\right)+\mathcal{D}_{B}\left(\mathcal{M}_{\left\{\widetilde{\Pi}_{i}^{A}\right\}}\left(\rho_{A B}\right)\right) \simeq 0.802628$.

Let us note that equation (17) can be rewritten in the following form via equations (11c), (14c) and (12)

$$
Q\left(\rho_{A B}\right)=\mathcal{I}\left(\rho_{A B}\right)-\mathcal{C}_{B}\left(\mathcal{M}_{\left\{\widetilde{\Pi}_{i}^{A}\right\}}\left(\rho_{A B}\right)\right),
$$

where equation (14c) was applied to the state $\mathcal{M}_{\left\{\widetilde{\Pi}_{i}^{A}\right\}}\left(\rho_{A B}\right)$. Therefore, we see that the overall bipartite classical correlations are given by

$$
C\left(\rho_{A B}\right)=\mathcal{C}_{B}\left(\mathcal{M}_{\left\{\widetilde{\Pi}_{i}^{A}\right\}}\left(\rho_{A B}\right)\right) .
$$

From equation (17) it follows that in general case the quantum discord $\mathcal{D}_{A}\left(\rho_{A B}\right)$ underestimates the bipartite quantum correlations, $\mathcal{D}_{A}\left(\rho_{A B}\right) \leq Q\left(\rho_{A B}\right)$. In other words the quantum discord $\mathcal{D}_{A}\left(\rho_{A B}\right)$ is a lower bound for the overall quantum correlations present in a bipartite state $\rho_{A B}$. From the other hand, the Henderson-Vedral measure of classical correlations, $\mathcal{C}_{A}\left(\rho_{A B}\right)$, overestimates the bipartite classical correlations because

$$
\begin{aligned}
\mathcal{C}_{A}\left(\rho_{A B}\right) & =\mathcal{I}\left(\rho_{A B}\right)-\mathcal{D}_{A}\left(\rho_{A B}\right) \\
& \geq \mathcal{I}\left(\rho_{A B}\right)-Q\left(\rho_{A B}\right)=C\left(\rho_{A B}\right),
\end{aligned}
$$

which means that $\mathcal{C}_{A}\left(\rho_{A B}\right)$ is an upper bound for the overall classical correlations present in a bipartite state $\rho_{A B}$. Let us note that $C\left(\rho_{A B}\right)$ can be rewritten, via equations (19), (16) and (15), in the form which coincides with the measure of classical correlations proposed in 36 .

$$
\begin{aligned}
C\left(\rho_{A B}\right) & =\mathcal{I}\left(\mathcal{M}_{\left\{\widetilde{\Pi}_{j}^{B}\right\}}\left(\mathcal{M}_{\left\{\widetilde{\Pi}_{i}^{A}\right\}}\left(\rho_{A B}\right)\right)\right) \\
& =H\left(\tilde{p}_{i}^{A}\right)+H\left(\tilde{p}_{j}^{B}\right)-H\left(\tilde{p}_{i j}^{A B}\right)=\mathcal{I}\left(\tilde{p}_{i j}^{A B}\right),
\end{aligned}
$$

where $\mathcal{I}\left(\tilde{p}_{i j}^{A B}\right)$ is the classical mutual information for the joint probability distribution $\tilde{p}_{i j}^{A B}$. Taking this into account we can rewrite $Q\left(\rho_{A B}\right)$, via equations (18) and (19), as follows

$$
Q\left(\rho_{A B}\right)=\mathcal{I}\left(\rho_{A B}\right)-\mathcal{I}\left(\tilde{p}_{i j}^{A B}\right),
$$

which shows explicitly that the measure of the overall quantum correlations $Q\left(\rho_{A B}\right)$ is symmetric with respect to the systems $A$ and $B$, because both mutual informations $\mathcal{I}\left(\rho_{A B}\right)$ and $\mathcal{I}\left(\tilde{p}_{i j}^{A B}\right)$ are symmetric.

Let us note finally that the above results shed new light on some recent developments and help to better understand them. Recently, it was numerically verified that for two-qubit states with maximally mixed reduced states, $\rho_{A}=\rho_{B}=\frac{1}{2} I$, we have $\mathcal{D}_{A}\left(\rho_{A B}\right)=Q\left(\rho_{A B}\right)$ [22]. In the framework of our approach, this result can be obtained analytically. It follows directly, via equation (17), from the fact that for these states $\mathcal{D}_{B}\left(\mathcal{M}_{\left\{\widetilde{\Pi}_{i}^{A}\right\}}\left(\rho_{A B}\right)\right)=0$, because the states $\rho_{B \mid i}$ in equation (13) commute as one can easily check. More recently, it has been reported that a zero-discord two-qubit $X$-state can have quantum correlations [37]. This result can be easily explained in the framework of our approach. In particular, from equation (17) it follows immediately that the nullity of quantum discord does not necessarily imply the vanishing of quantum correlations.

\section{CORRELATIONS IN MULTIPARTITE SYSTEMS}

In this section, we will show that a notion of quantum discord can be extended in a natural way to multipartite quantum systems by invoking quantum relative entropy. Then, we will find the overall quantum and classical correlations present in these systems.

The quantum relative entropy of a state $\rho$ with respect to a state $\sigma$ is defined as $S(\rho \| \sigma)=-S(\rho)-\operatorname{Tr}\left(\rho \log _{2} \sigma\right)$. The quantum mutual information (1) is only a special case of quantum relative entropy, namely it is the quantum relative entropy of $\rho_{A B}$ with respect to $\rho_{A} \otimes \rho_{B}$, $\mathcal{I}\left(\rho_{A B}\right)=S\left(\rho_{A B} \| \rho_{A} \otimes \rho_{B}\right)$ (see e.g., 34]). Therefore, in this way we can naturally generalize a notion of quantum mutual information to multipartite systems and thereby a notion of quantum discord via quantum relative entropy.

Let us consider $m$ quantum systems, $A_{1} \ldots A_{m}$, in a state $\rho_{\mathbf{A}}$. The quantum mutual information of a state $\rho_{\mathbf{A}}$ is given by

$$
\begin{aligned}
\mathcal{I}\left(\rho_{\mathbf{A}}\right) & =S\left(\rho_{\mathbf{A}} \| \rho_{A_{1}} \otimes \cdots \otimes \rho_{A_{m}}\right) \\
& =\sum_{i} S\left(\rho_{A_{i}}\right)-S\left(\rho_{\mathbf{A}}\right),
\end{aligned}
$$

which allows us to define quantum discord for a $m$-partite system.

The quantum conditional entropy, $S\left(\rho_{\left[A_{k}\right] \mid A_{k}}\right)=$ $S\left(\rho_{\mathbf{A}}\right)-S\left(\rho_{A_{k}}\right)$, allows one to rewrite the quantum mutual information in the following form

$$
\mathcal{I}\left(\rho_{\mathbf{A}}\right)=\sum_{i \neq k} S\left(\rho_{A_{i}}\right)-S\left(\rho_{\left[A_{k}\right] \mid A_{k}}\right),
$$

where $\left[A_{k}\right]$ stands for $A_{1} \ldots A_{k-1} A_{k+1} \ldots A_{m}$. The fact that the quantum conditional entropy quantifies the ignorance about the systems $\left[A_{k}\right]$ that remains if we make measurements on the system $A_{k}$ allows one to find an alternative expression for the quantum conditional entropy, and thereby for the quantum mutual information.

If the von Neumann projective measurement, $\left\{\Pi_{i}^{A_{k}}\right\}$, corresponding to outcomes $i$, is performed then the post- 
measurement joint state of the systems $\left[A_{k}\right]$ is given by

$$
\rho_{\left[A_{k}\right] \mid i}=\operatorname{Tr}_{A_{k}}\left[\mathcal{P}_{i}^{A_{k}} \rho_{\mathbf{A}} \mathcal{P}_{i}^{A_{k}}\right] / p_{i}^{A_{k}}
$$

where $\mathcal{P}_{i}^{A_{k}}=\left(I \otimes \cdots \otimes \Pi_{i}^{A_{k}} \otimes \cdots \otimes I\right)$ and $p_{i}^{A_{k}}=$ $\operatorname{Tr}\left[\mathcal{P}_{i}^{A_{k}} \rho_{\mathbf{A}}\right]$. The von Neumann entropies $S\left(\rho_{\left[A_{k}\right] \mid i}\right)$, weighted by probabilities $p_{i}^{A_{k}}$, lead to the quantum conditional entropy of the systems $\left[A_{k}\right]$ given the complete measurement $\left\{\Pi_{i}^{A_{k}}\right\}$ on the system $A_{k}$

$$
S_{\left\{\Pi_{i}^{A_{k}}\right\}}\left(\rho_{\left[A_{k}\right] \mid i}\right)=\sum_{i} p_{i}^{A_{k}} S\left(\rho_{\left[A_{k}\right] \mid i}\right),
$$

and thereby the quantum mutual information, induced by the von Neumann measurement performed on the system $A_{k}$, is defined by

$$
\mathcal{J}_{\left\{\Pi_{i}^{A_{k}}\right\}}\left(\rho_{\mathbf{A}}\right)=\sum_{i \neq k} S\left(\rho_{A_{i}}\right)-S_{\left\{\Pi_{i}^{A_{k}}\right\}}\left(\rho_{\left[A_{k}\right] \mid A_{k}}\right) .
$$

The measurement independent quantum mutual information $\mathcal{J}_{A_{k}}\left(\rho_{\mathbf{A}}\right)$ is defined by

$$
\begin{aligned}
\mathcal{J}_{A_{k}}\left(\rho_{\mathbf{A}}\right)= & \sup _{\left\{\Pi_{i}^{A_{k}}\right\}} \mathcal{J}_{\left\{\Pi_{i}^{A_{k}}\right\}}\left(\rho_{\mathbf{A}}\right) \\
= & \sum_{i \neq k} S\left(\rho_{A_{i}}\right)-\inf _{\left\{\Pi_{i}^{A_{k}}\right\}} \sum_{i} p_{i}^{A_{k}} S\left(\rho_{\left[A_{k}\right] \mid i}\right) .
\end{aligned}
$$

Therefore, we define the quantum discord $\mathcal{D}_{A_{k}}\left(\rho_{\mathbf{A}}\right)$ as follows

$$
\begin{aligned}
\mathcal{D}_{A_{k}}\left(\rho_{\mathbf{A}}\right) & =\mathcal{I}\left(\rho_{\mathbf{A}}\right)-\mathcal{J}_{A_{k}}\left(\rho_{\mathbf{A}}\right) \\
& =S\left(\rho_{A_{k}}\right)-S\left(\rho_{\mathbf{A}}\right) \\
& +\inf _{\left\{\Pi_{i}^{A_{k}}\right\}} \sum_{i} p_{i}^{A_{k}} S\left(\rho_{\left[A_{k}\right] \mid i}\right) .
\end{aligned}
$$

Thus, $\mathcal{J}_{A_{k}}\left(\rho_{\mathbf{A}}\right)$ can be interpreted as a measure of classical correlations

$$
\mathcal{C}_{A_{k}}\left(\rho_{\mathbf{A}}\right)=\sum_{i \neq k} S\left(\rho_{A_{i}}\right)-\inf _{\left\{\Pi_{i}^{A_{k}}\right\}} \sum_{i} p_{i}^{A_{k}} S\left(\rho_{\left[A_{k}\right] \mid i}\right),
$$

and consequently

$$
\mathcal{D}_{A_{k}}\left(\rho_{\mathbf{A}}\right)=\mathcal{I}\left(\rho_{\mathbf{A}}\right)-\mathcal{C}_{A_{k}}\left(\rho_{\mathbf{A}}\right)
$$

Of course, the quantum discord $\mathcal{D}_{A_{k}}\left(\rho_{\mathbf{A}}\right)$ can be expressed alternatively as the minimal loss of correlations caused by the non-selective von Neumann projective measurement performed on the system $A_{k}$

$$
\mathcal{D}_{A_{k}}\left(\rho_{\mathbf{A}}\right)=\inf _{\left\{\Pi_{i}^{A_{k}}\right\}}\left[\mathcal{I}\left(\rho_{\mathbf{A}}\right)-\mathcal{I}\left(\mathcal{M}_{\left\{\Pi_{i}^{A_{k}}\right\}}\left(\rho_{\mathbf{A}}\right)\right],\right.
$$

where $\mathcal{M}_{\left\{\Pi_{i}^{A_{k}}\right\}}\left(\rho_{\mathbf{A}}\right)=\sum_{i} \mathcal{P}_{i}^{A_{k}} \rho_{\mathbf{A}} \mathcal{P}_{i}^{A_{k}}$. Obviously, the optimal measurement $\mathcal{M}_{\left\{\widetilde{\Pi}_{i}^{A_{k}}\right\}}$, for which infimum in equation (32) is attained, causes only the loss of quantum correlations.
We can now use the above considerations to investigate quantum correlations present in a state $\rho_{\mathbf{A}}$. Let us assume that the optimal non-selective von Neumann projective measurements $\mathcal{M}_{\left\{\widetilde{\Pi}_{i}^{A_{1}}\right\}}, \ldots, \mathcal{M}_{\left\{\widetilde{\Pi}_{i}^{A_{m}}\right\}}$ leading to the minimal loss of quantum correlations are performed subsequently on $m$ quantum systems $A_{1} \ldots A_{m}$. Clearly, the corresponding post-measurement states are given by

$$
\begin{aligned}
& \mathcal{M}_{\left\{\widetilde{\Pi}_{i_{1}}^{A_{1}}\right\}}\left(\rho_{\mathbf{A}}\right), \\
& \mathcal{M}_{\left\{\widetilde{\Pi}_{i_{2}}^{A_{2}}\right\}}\left(\mathcal{M}_{\left\{\widetilde{\Pi}_{i_{1}}^{A_{1}}\right\}}\left(\rho_{\mathbf{A}}\right)\right), \\
& \vdots \\
& \mathcal{M}_{\left\{\widetilde{\Pi}_{i_{m}}^{A_{m}}\right\}}\left(\ldots\left(\mathcal{M}_{\left\{\widetilde{\Pi}_{i_{1}}^{A_{1}}\right\}}\left(\rho_{\mathbf{A}}\right)\right)\right) .
\end{aligned}
$$

Each of these states can have quantum correlations, except the last one which has only classical correlations. Therefore, the subsequent measurements lead to the corresponding loss of quantum correlations

$$
\begin{aligned}
& \mathcal{D}_{A_{1}}\left(\rho_{\mathbf{A}}\right) \\
& \mathcal{D}_{A_{2}}\left(\mathcal{M}_{\left\{\widetilde{\Pi}_{i_{1}}^{A_{1}}\right\}}\left(\rho_{\mathbf{A}}\right)\right), \\
& \mathcal{D}_{A_{3}}\left(\mathcal{M}_{\left\{\widetilde{\Pi}_{i_{2}}^{A_{2}}\right\}}\left(\mathcal{M}_{\left\{\widetilde{\Pi}_{i_{1}}^{\left.A_{1}\right\}}\right.}\left(\rho_{\mathbf{A}}\right)\right)\right) \\
& \vdots \\
& \mathcal{D}_{A_{m}}\left(\mathcal{M}_{\left\{\widetilde{\Pi}_{i_{m-1}}^{A_{m-1}}\right\}}\left(\ldots\left(\mathcal{M}_{\left\{\widetilde{\Pi}_{i_{1}}^{A_{1}}\right\}}\left(\rho_{\mathbf{A}}\right)\right)\right)\right) .
\end{aligned}
$$

Therefore, the overall quantum correlations present in a $m$-partite quantum state $\rho_{\mathbf{A}}$ are measured by

$$
\begin{aligned}
Q\left(\rho_{\mathbf{A}}\right) & =\mathcal{D}_{A_{1}}\left(\rho_{\mathbf{A}}\right)+\mathcal{D}_{A_{2}}\left(\mathcal{M}_{\left\{\widetilde{\Pi}_{i_{1}}^{A_{1}}\right\}}\left(\rho_{\mathbf{A}}\right)\right) \\
& +\mathcal{D}_{A_{3}}\left(\mathcal{M}_{\left\{\widetilde{\Pi}_{i_{2}}^{A_{2}}\right\}}\left(\mathcal{M}_{\left\{\widetilde{\Pi}_{i_{1}}^{A_{1}}\right\}}\left(\rho_{\mathbf{A}}\right)\right)\right) \\
& +\cdots+\mathcal{D}_{A_{m}}\left(\mathcal{M}_{\left\{\widetilde{\Pi}_{i_{m}-1}^{A_{m}-1}\right\}}\left(\ldots\left(\mathcal{M}_{\left\{\widetilde{\Pi}_{i_{1}}^{A_{1}}\right\}}\left(\rho_{\mathbf{A}}\right)\right)\right)\right),
\end{aligned}
$$

which is a multipartite generalization of the measure (17) introduced in the previous section. This equation can be rewritten, via equation (31) and due to the fact that each measurement remains classical correlations unaffected, in the following form

$$
Q\left(\rho_{\mathbf{A}}\right)=\mathcal{I}\left(\rho_{\mathbf{A}}\right)-\mathcal{C}_{A_{m}}\left(\mathcal{M}_{\left\{\widetilde{\Pi}_{i_{m-1}}^{A_{m-1}}\right\}}\left(\ldots\left(\mathcal{M}_{\left\{\widetilde{\Pi}_{i_{1}}^{A_{1}}\right\}}\left(\rho_{\mathbf{A}}\right)\right)\right)\right) .
$$

Therefore, the overall multipartite classical correlations are given by

$$
C\left(\rho_{\mathbf{A}}\right)=\mathcal{C}_{A_{m}}\left(\mathcal{M}_{\left\{\widetilde{\Pi}_{i_{m-1}}^{A_{m-1}}\right\}}\left(\ldots\left(\mathcal{M}_{\left\{\widetilde{\Pi}_{i_{1}}^{A_{1}}\right\}}\left(\rho_{\mathbf{A}}\right)\right)\right)\right) .
$$

From equations (31) and it follows that $C\left(\rho_{\mathbf{A}}\right)$ can be rewritten as

$$
\begin{aligned}
C\left(\rho_{\mathbf{A}}\right) & =\mathcal{I}\left(\mathcal{M}_{\left\{\widetilde{\Pi}_{i_{m}}^{A_{m}}\right\}}\left(\ldots\left(\mathcal{M}_{\left\{\widetilde{\Pi}_{i_{1}}^{A_{1}}\right\}}\left(\rho_{\mathbf{A}}\right)\right)\right)\right) \\
& =\mathcal{I}\left(\sum_{i_{1} \ldots i_{m}} \tilde{p}_{i_{1} \ldots i_{m}}^{A_{1} \ldots A_{m}} \widetilde{\Pi}_{i_{1}}^{A_{1}} \otimes \cdots \otimes \widetilde{\Pi}_{i_{m}}^{A_{m}}\right)
\end{aligned}
$$


where $\tilde{p}_{i_{1} \ldots i_{m}}^{A_{1} \ldots A_{m}}=\operatorname{Tr}\left[\left(\widetilde{\Pi}_{i_{1}}^{A_{1}} \otimes \cdots \otimes \widetilde{\Pi}_{i_{m}}^{A_{m}}\right) \rho_{\mathbf{A}}\right]$. From equation (23) it follows that

$$
C\left(\rho_{\mathbf{A}}\right)=\sum_{k=1}^{m} H\left(\tilde{p}_{i_{k}}^{A_{k}}\right)-H\left(\tilde{p}_{i_{1} \ldots i_{m}}^{A_{1} \ldots A_{m}}\right)=\mathcal{I}\left(\tilde{p}_{i_{1} \ldots i_{m}}^{A_{1} \ldots A_{m}}\right),
$$

where $\mathcal{I}\left(\tilde{p}_{i_{1} \ldots i_{m}}^{A_{1} \ldots A_{m}}\right)$ is the classical mutual information for the joint probability distribution $\tilde{p}_{i_{1} \ldots i_{m}}^{A_{1} \ldots A_{m}}$. Therefore, the overall multipartite quantum correlations can be rewritten in the following form

$$
Q\left(\rho_{\mathbf{A}}\right)=\mathcal{I}\left(\rho_{\mathbf{A}}\right)-\mathcal{I}\left(\tilde{p}_{i_{1} \ldots i_{m}}^{A_{1} \ldots A_{m}}\right) .
$$

Let us note that the nullity of the quantum discord $\mathcal{D}_{A_{1}}\left(\rho_{\mathbf{A}}\right)$ does not necessarily imply the vanishing of quantum correlations present in a multipartite state, because the quantum discord $\mathcal{D}_{A_{1}}\left(\rho_{\mathbf{A}}\right)$ is a lower bound for the overall multipartite quantum correlations. Obviously, multipartite counterpart of the Henderson-Vedral measure of classical correlations, $\mathcal{C}_{A_{1}}\left(\rho_{\mathbf{A}}\right)$, is an upper bound for the overall multipartite classical correlations.

\section{SUMMARY}

Using the alternative formulation of quantum discord, we have provided a systematical analysis of quantum and classical correlations present in bipartite quantum systems. In particular, we have introduced a new measure of the overall quantum correlations, and showed that this measure is lower bounded by quantum discord. This implies that a zero-discord state can have quantum correlations. Finally, we have shown that our approach to quantification of correlations can be naturally extended to multipartite quantum systems.

\section{Acknowledgments}

This work was supported by the University of Lodz Grant, the Polish Ministry of Science and Higher Education Grant No. N N202 103738, and the Polish Research Network Laboratory of Physical Foundations of Information Processing. M. Okrasa acknowledges the support from the European Union under the Human Capital Programme - Measure 8.2.1 and the IROP - Measure 2.6.
[1] R. Horodecki, P. Horodecki, M. Horodecki, and K. Horodecki, Rev. Mod. Phys. 81, 865 (2009).

[2] O. Gühne and G. Tóth, Phys. Rep. 474, 1 (2009).

[3] R. F. Werner, Phys. Rev. A 40, 4277 (1989).

[4] E. Knill and R. Laflamme, Phys. Rev. Lett. 81, 5672 (1998).

[5] S. L. Braunstein, C. M. Caves, R. Jozsa, N. Linden, S. Popescu, and R. Schack, Phys. Rev. Lett. 83, 1054 (1999).

[6] C. H. Bennett, D. P. DiVincenzo, C. A. Fuchs, T. Mor, E. Rains, P. W. Shor, J. A. Smolin, and W. K. Wootters, Phys. Rev. A 59, 1070 (1999).

[7] D. A. Meyer, Phys. Rev. Lett. 85, 2014 (2000).

[8] E. Biham, G. Brassard, D. Kenigsberg, and T. Mor, Theor. Comput. Sci. 320, 15 (2004).

[9] A. Datta, S. T. Flammia, and C. M. Caves, Phys. Rev. A 72, 042316 (2005).

[10] A. Datta and G. Vidal, Phys. Rev. A 75, 042310 (2007).

[11] H. Ollivier and W. H. Zurek, Phys. Rev. Lett. 88, 017901 (2001).

[12] L. Henderson and V. Vedral, J. Phys. A 34, 6899 (2001).

[13] A. Datta, A. Shaji, and C. M. Caves, Phys. Rev. Lett. 100, 050502 (2008).

[14] B. P. Lanyon, M. Barbieri, M. P. Almeida, and A. G. White, Phys. Rev. Lett. 101, 200501 (2008).

[15] A. Datta and S. Gharibian, Phys. Rev. A 79, 042325 (2009).

[16] S. Luo, Phys. Rev. A 77, 042303 (2008).
[17] M. Ali, A. R. P. Rau, and G. Alber, Phys. Rev. A 81, 042105 (2010).

[18] M. D. Lang and C. M. Caves, Phys. Rev. Lett. 105, 150501 (2010).

[19] T. Werlang, S. Souza, F. F. Fanchini, and C. J. Villas Boas, Phys. Rev. A 80, 024103 (2009).

[20] J. Maziero, L. C. Céleri, R. M. Serra, and V. Vedral, Phys. Rev. A 80, 044102 (2009).

[21] F. F. Fanchini, T. Werlang, C. A. Brasil, L. G. E. Arruda, and A. O. Caldeira, Phys. Rev. A 81, 052107 (2010).

[22] J. Maziero, T. Werlang, F. F. Fanchini, L. C. Céleri, and R. M. Serra, Phys. Rev. A 81, 022116 (2010).

[23] B. Wang, Z.-Y. Xu, Z.-Q. Chen, and M. Feng, Phys. Rev. A 81, 014101 (2010).

[24] C. A. Rodríguez-Rosario, K. Modi, A. Kuah, A. Shaji, and E. C. G. Sudarshan, J. Phys. A 41, 205301 (2008).

[25] A. Shabani and D. A. Lidar, Phys. Rev. Lett. 102, 100402 (2009).

[26] M. Piani, P. Horodecki, and R. Horodecki, Phys. Rev. Lett. 100, 090502 (2008).

[27] A. Ferraro, L. Aolita, D. Cavalcanti, F. M. Cucchietti, and A. Acín, Phys. Rev. A 81, 052318 (2010).

[28] B. Dakić, V. Vedral, and Č. Brukner, Phys. Rev. Lett. 105, 190502 (2010).

[29] B. Bylicka and D. Chruściński, Phys. Rev. A 81, 062102 (2010).

[30] P. Giorda and M. G. A. Paris, Phys. Rev. Lett. 105, 020503 (2010). 
[31] G. Adesso and A. Datta, Phys. Rev. Lett. 105, 030501 (2010).

[32] S. Luo and S. Fu, Phys. Rev. A 82, 034302 (2010).

[33] K. Kraus, States, Effects and Operations: Fundamental Notions of Quantum Theory (Springer-Verlag, Berlin, 1983).

[34] M. A. Nielsen and I. L. Chuang, Quantum Computation and Quantum Information (Cambridge University Press, Cambridge, 2000).

[35] A. Datta, arXiv:0807.4490.

[36] B. M. Terhal, M. Horodecki, D. W. Leung, and D. P. DiVincenzo, J. Math. Phys. 43, 4286 (2002).

[37] G.-X. Li, Z. Yi, and Z. Ficek, arXiv:1101.4983. 